Supporting Information for

\title{
Construction of a Carbon Dots/Cobalt Oxyhydroxide Nanoflakes Biosensing Platform for Detection of Acid Phosphatase
}

\author{
Haijuan Zhang ${ }^{\dagger, \$, \#}$, Yangxia Han ${ }^{\dagger, \#}$, Yali Yang ${ }^{\dagger}$, Jia Chen ${ }^{\dagger}$, Hongdeng Qiu ${ }^{\dagger, \S, \&, *}$
}

${ }^{\dagger}$ CAS Key Laboratory of Chemistry of Northwestern Plant Resources and Key Laboratory for Natural Medicine of Gansu Province, Lanzhou Institute of Chemical Physics, Chinese Academy of Sciences, Lanzhou 730000, China

$\$$ State Key Laboratory of Grassland Agro-Ecosystem, Institute of Innovation Ecology, Lanzhou University, Lanzhou 730000, China

$\S$ College of Chemistry, Zhengzhou University, Zhengzhou 450001, China

\& School of Chemistry and Chemical Engineering, Gannan Normal University, Ganzhou 341000, China

${ }^{\#}$ H. Zhang and Y. Han contributed equally to this work.

* Corresponding E-mail: hdqiu @licp.cas.cn (H. Qiu) 


\section{Reagents and Apparatuses}

p-Phenylenediamine (PPD), PEG 1000, $\mathrm{NaClO}, \mathrm{CoCl}_{2}, \mathrm{NaOH}$, ascorbic acid (AA) were bought from Sigma-Aldrich Corporation, ascorbic acid-2-phosphate trisodium salt (AAP) was obtained from Meryer (Shanghai) Chemical Technology Co., Ltd., acid phosphatase (ACP) was purchased from HUAAOBIO (Beijing) Technology Co., Ltd., $20 \mathrm{mM}$ PBS $\left(\mathrm{Na}_{2} \mathrm{HPO}_{4}-\mathrm{NaH}_{2} \mathrm{PO}_{4}\right)$ was formulated with the deionized water.

Fluorescence data were obtained from a Fluoromax-4 spectrofluorometer. The ultraviolet-visible absorption spectra were collected on a PerkinElmer Lambda 35 ultraviolet-visible spectrometer (UV-vis). Transmission electron microscopy (TEM) images of the RCDs were obtained from a Tecnai G2 TF20 instrument. Powder X-ray diffraction (XRD) pattern was collected on a XRD-6100 X-ray diffractometer with $\mathrm{Cu} \mathrm{K} \alpha$ radiation. Fourier transform infrared spectrum (FT-IR) was conducted on a Nexus 870 spectrometer using KBr pellets. 
A

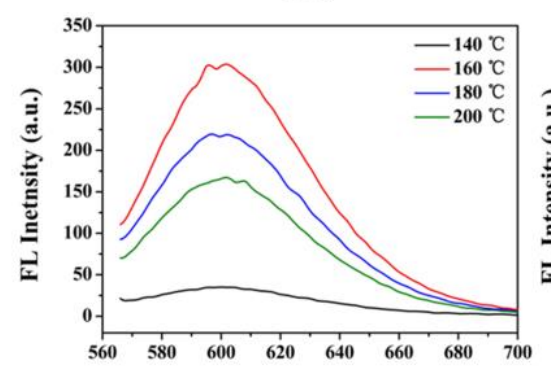

Wavelength (nm)

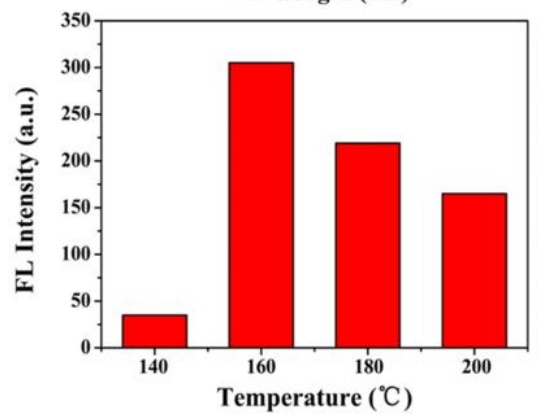

B
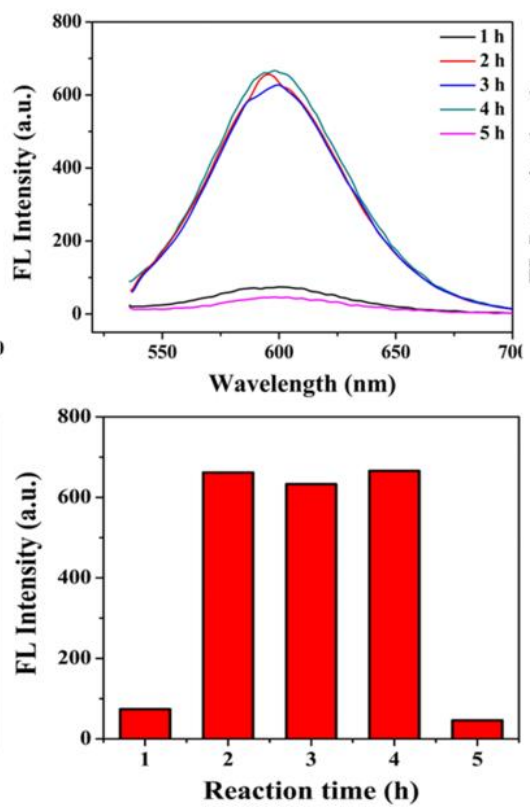

C
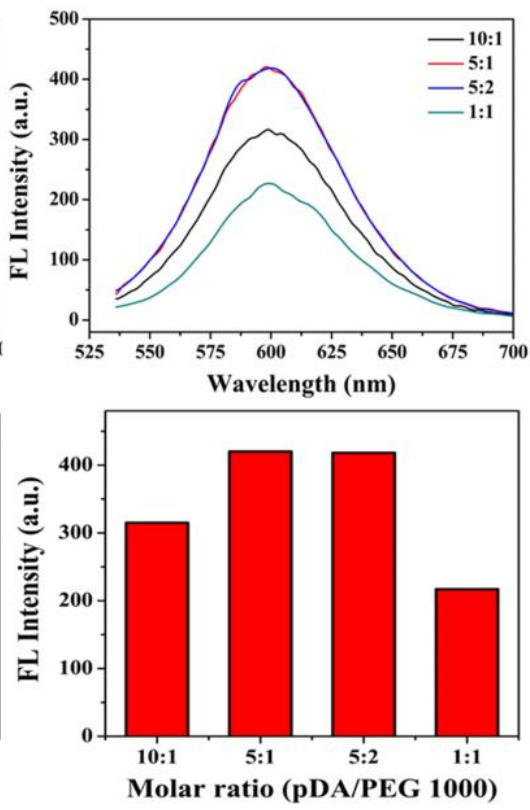

Figure S1. Fluorescent spectra and FL intensities of the RCDs obtained under different carbonization conditions: (A) temperature, (B) time $\left(\mathrm{T}=160^{\circ} \mathrm{C}\right),(\mathrm{C})$ molar ratio $\left(\mathrm{T}=160^{\circ} \mathrm{C}, \mathrm{t}=2 \mathrm{~h}\right)$. 

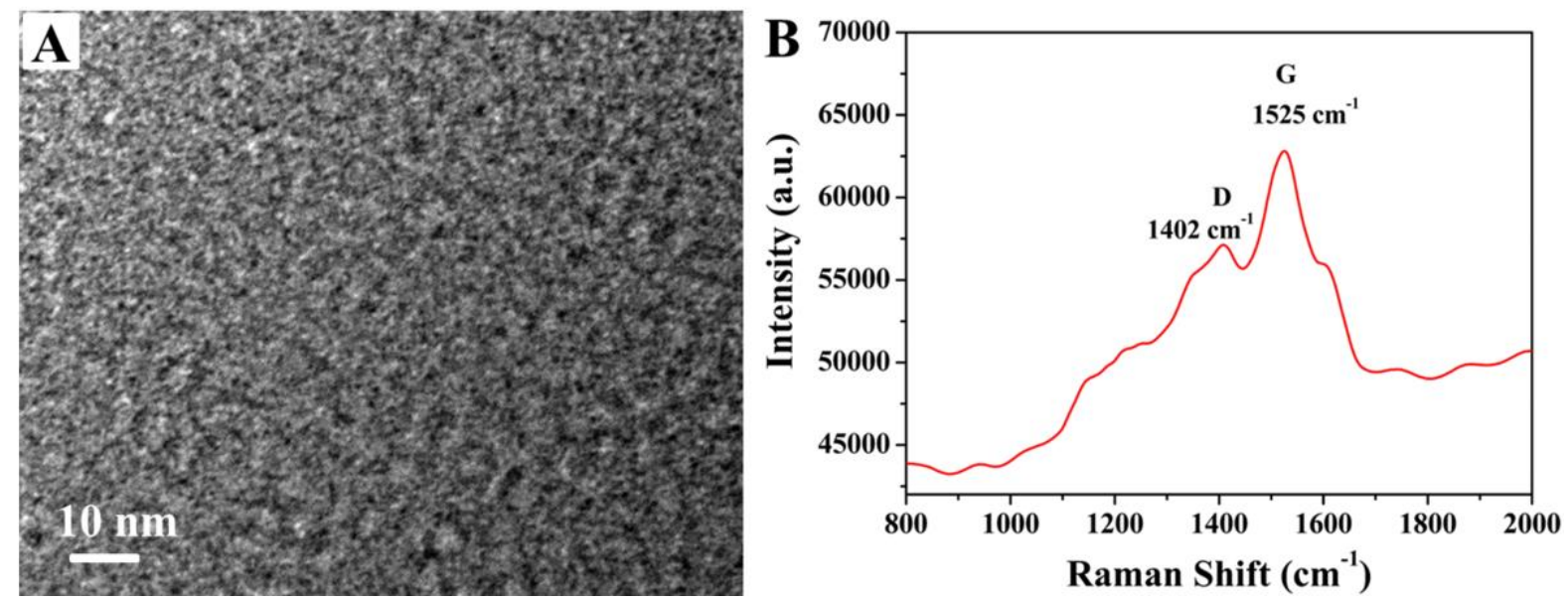

Figure S2. (A) HRTEM image and (B) Raman spectrum of the RCDs. 


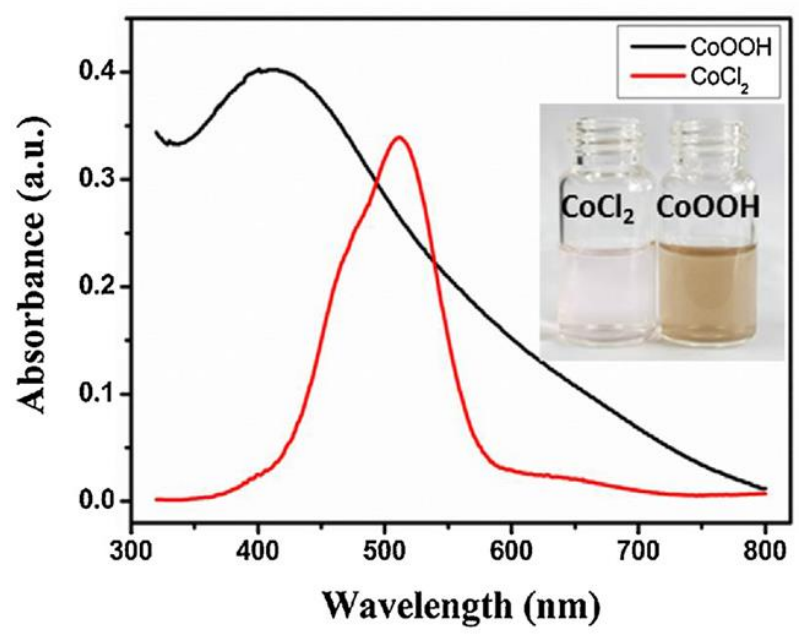

Figure S3. UV-vis spectra of the $\mathrm{CoCl}_{2}$ and $\mathrm{CoOOH}$ nanoflakes. Inset were the pictures of the $\mathrm{CoCl}_{2}$ and $\mathrm{CoOOH}$ nanoflakes taken under visible light. 


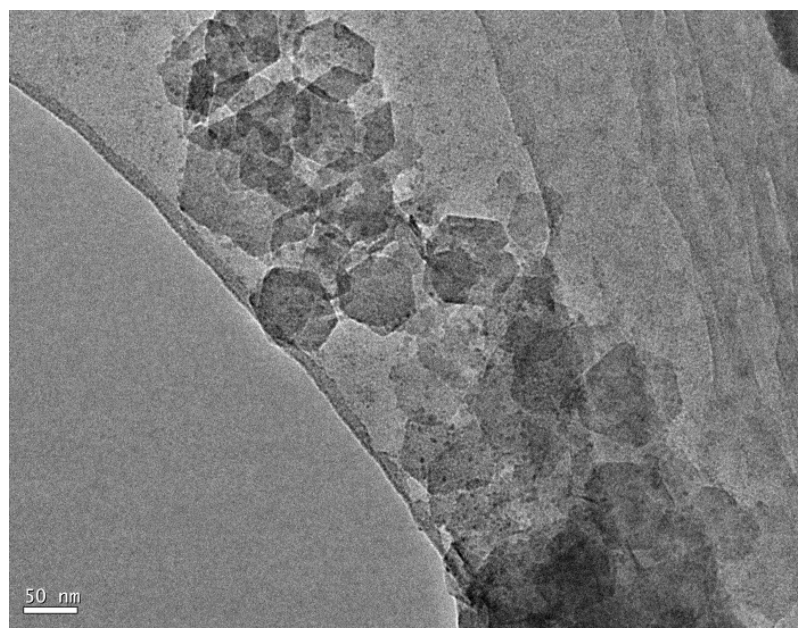

Figure S4. TEM image of the mixed system of RCDs and $\mathrm{CoOOH}$ nanoflakes. 


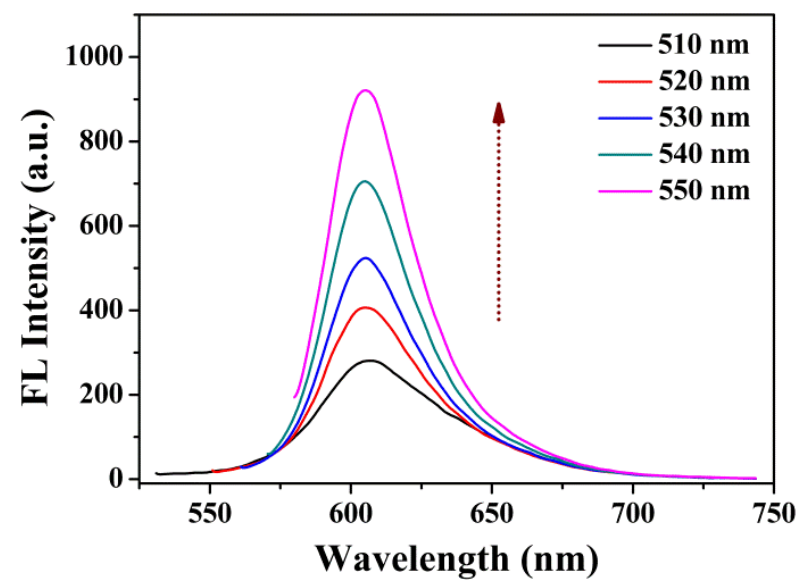

Figure S5. FL emission spectra of the RCDs with excitation wavelengths varied from 510 to 550 nm. 

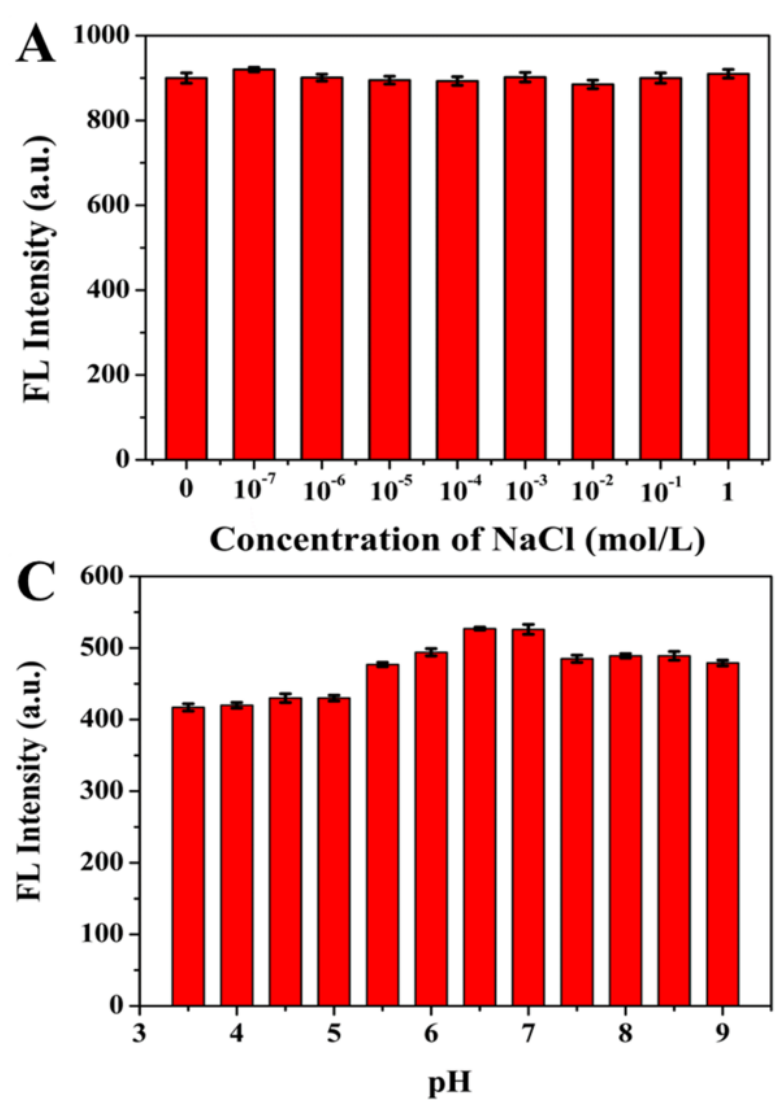

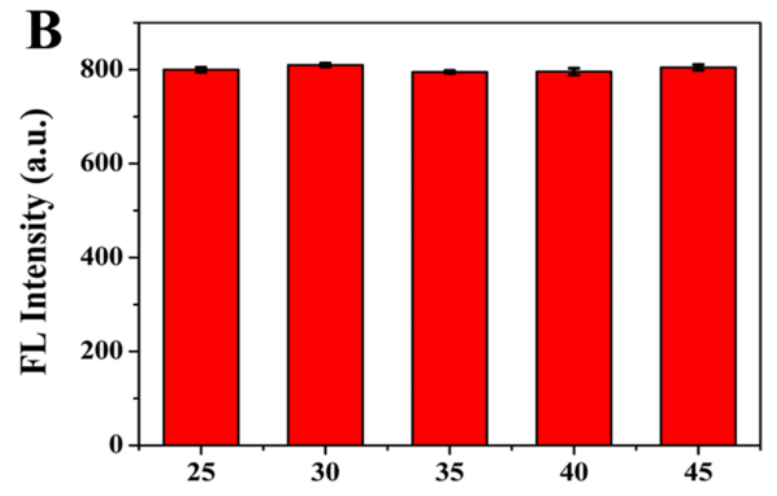

Temperature $\left({ }^{\circ} \mathrm{C}\right)$

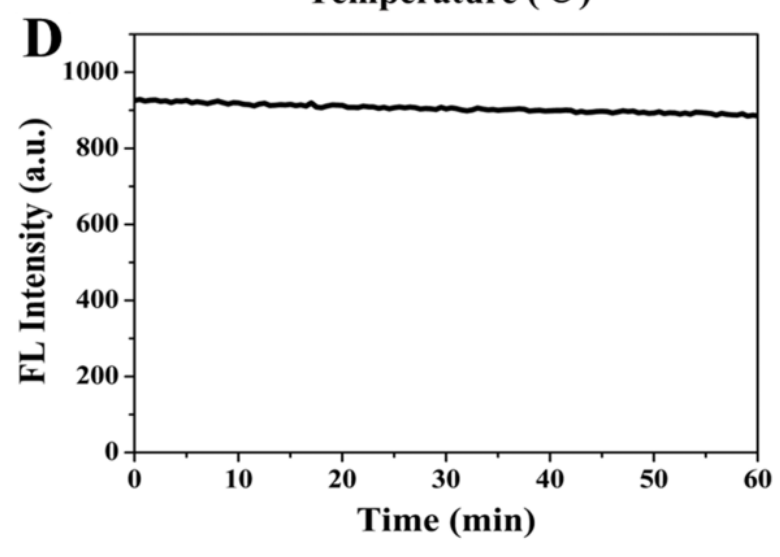

Figure S6. FL intensity variation of the RCDs as a function of (A) $\mathrm{NaCl}$ concentration, (B) temperature, $(\mathrm{C}) \mathrm{pH}$ values, and (D) light illumination $\left(\lambda_{\mathrm{ex}}=540 \mathrm{~nm}\right)$. 

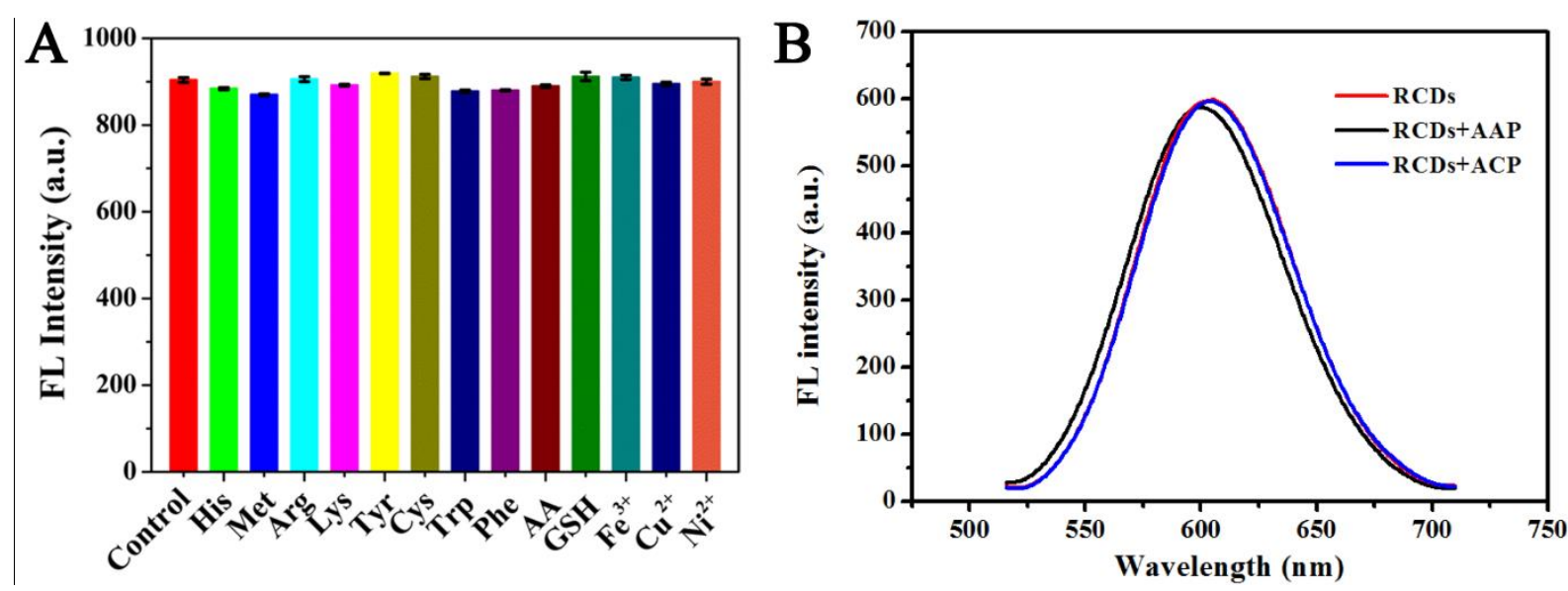

Figure S7. (A) Influences of various biological substances $(50 \mu \mathrm{mol} / \mathrm{L})$ on the FL intensity of the RCDs, (B) Influences of $200 \mu \mathrm{M}$ AAP and $16 \mu \mathrm{U} / \mathrm{mL}$ ACP on the FL intensity of the RCDs. 


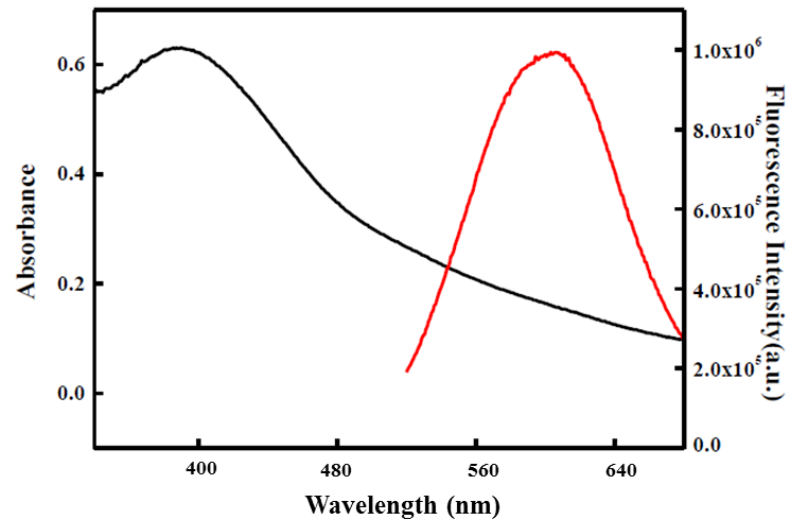

Figure S8. UV-vis absorption spectrum of $\mathrm{CoOOH}$ (black line) and the fluorescence emission spectrum of the RCDs (red line, $\lambda \mathrm{ex}=540 \mathrm{~nm}$ ). 


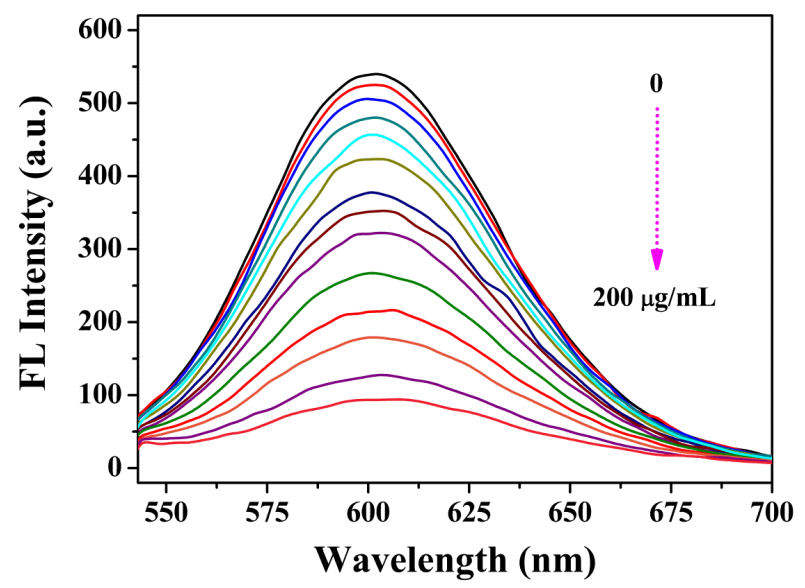

Figure S9. Fluorescence quenching of varied concentration of $\mathrm{CoOOH}$ nanoflakes on the fluorescence of the RCDs. 


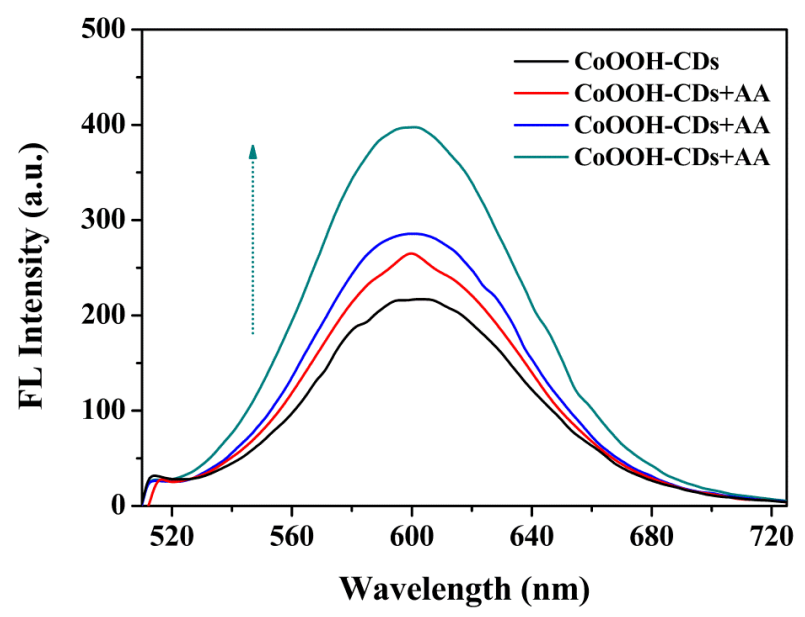

Figure S10. Fluorescence recovery of varied concentration of AA on the fluorescence of the RCDs (the concentration of AA from bottom to top was taken as 0,40, 80, and $200 \mu \mathrm{M}$ ). 


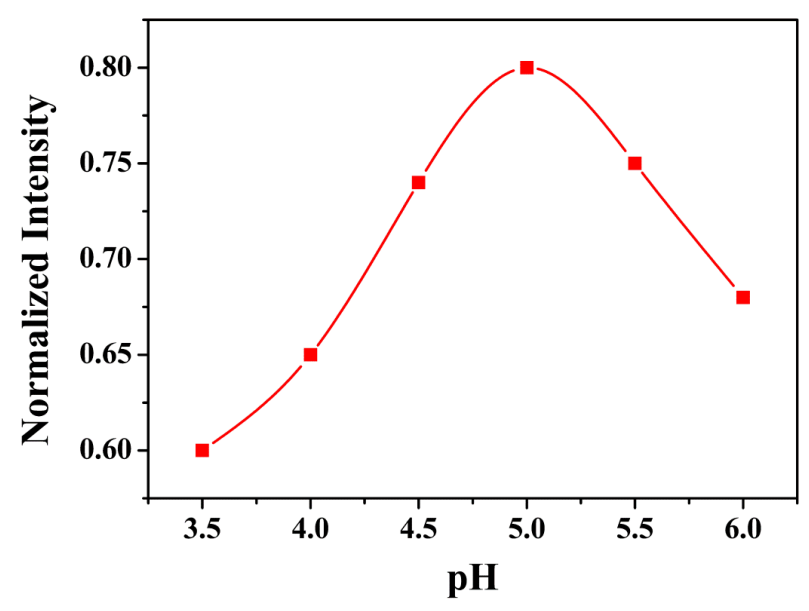

Figure S11. Influences of varied $\mathrm{pH}$ on the detection of $\mathrm{ACP}(16 \mu \mathrm{U} / \mathrm{mL})$. 


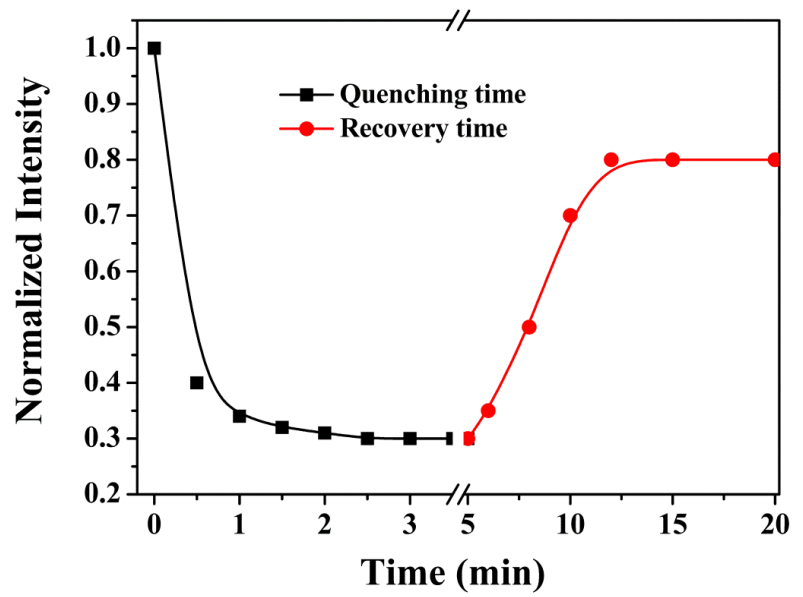

Figure S12. Influences of reaction time on the detection of ACP $(16 \mu \mathrm{U} / \mathrm{mL})$. 